\title{
Identification and assessment of the current scientific evidence on percutaneous needle electrolysis
}

\author{
Valera Garrido F. 1,2,3 Minaya Muñoz F. 1,2,3 Martínez Ramírez P. 1,2,3 Espinola Marcos A. 1,2,3 \\ ${ }^{1}$ MVClinic, Madrid, Spain \\ 2 Valencia Basket Club, Valencia, Spain \\ ${ }^{3}$ Centro de Fisioterapia Espinola, Valencia, Spain
}

Rev Fisioter Invasiva 2019;2:117-118.

\begin{abstract}
\section{Keywords}

- percutaneous needle electrolysis

- scientific evidence

Introduction Percutaneous needle electrolysis is an invasive physical therapy technique which was first published in a scientific journal in the year 2008. The aim of the present study was to evaluate the current scientific evidence on percutaneous needle electrolysis in neuro-musculoskeletal system disorders.

Material and Methods A retrospective observational study of scientific publications on percutaneous needle electrolysis. An online search was performed in the following databases to access abstracts: MEDLINE, PEDro, COCHRANE, IME, ENFISPO, in addition non-indexed journals were revised, using the following key words: [("percutaneous electrolysis," "galvanic electrolysis," "percutaneous direct current stimulation," "percutaneous microelectrolysis" and the Spanish terms "electrolisis," "electrolisis percutánea," "microelectrolisis")]. The search period ranged from the year the database was created until the time of the study (September 2018) with no other search limitation and including publications in any language. Furthermore, a manual search was performed based on the citations included in the articles. A first screen was performed based on the retrieved abstracts, and the full-text of each article was evaluated to analyze the scientific evidence of each study, based on the scale described by the US Preventive Task Force, which assigns level I to those studies with a greater quality design and scientific rigor, whereas level III is assigned to those of poorer quality. The assignment of the level of evidence of each study was independently and simultaneously evaluated by two observers with no communication between each other. To determine reliability, the Kappa index was used $(\mathrm{K})$ with a $95 \% \mathrm{Cl}$. The score reached by the clinical trials and systematic reviews was also revised based on the Pedro Score Database.

Results In total, 64 documents were identified related with electrolysis, following the PRISMA recommendations for the search and selection of primary studies. Concretely, 39 papers were retrieved directly from database searches (Medline, PEDro, Cochrane, ENFISPO), whereas 25 were indirectly accessed via other sources based on internet searches and citation searches. Eight papers were excluded after filtering by title and abstract. After reading the full-text of the 56 articles selected, one further article was excluded. Finally, 55 articles were included in the review. The evidence of the studies retrieved was evaluated. The reliability analysis obtained an acceptable Kappa index
\end{abstract}

Copyright $\odot 2019$ by Thieme Revinter Publicações Ltda, Rio de Janeiro, Brazil 
(0.6-0.8). Based on criteria regarding the quality of evidence, the studies were classified into three groups:

a) High-level of evidence. This group included clinical trials (of which there were 9 , which constitute $16.7 \%$ of the total) $(77.8 \%$ were published after 2017 ).

b) Medium-level of evidence, identified in $35.2 \%$ of papers.

c) Low evidence was provided in $48.1 \%$ of papers.

Conclusions In recent years, there has been a considerable increase in the current evidence available on percutaneous needle electrolysis. However, many of these studies are part of the gray literature and are difficult to access. High quality evidence was identified in $16.7 \%$ of papers. The remaining $83.3 \%$ papers were of low to medium quality. 